\title{
Die 59. Internationale Mathematik-Olympiade
}

\author{
Jürgen Prestin
}

Die 59. Internationale Mathematik-Olympiade fand vom 3. bis zum 14. Juli 2018 in Cluj-Napoca, der zweitgrößten Stadt Rumäniens, statt.

6o Schülerinnen und 534 Schüler aus 107 Ländern nahmen an dieser Olympiade teil.

Die deutsche Mannschaft bestand aus sechs Schülern, Dr. Christian Reiher als stellvertretendem Delegationsleiter und dem Berichterstatter als Delegationsleiter.

Tabelle 1. Das deutsche Team

\begin{tabular}{|c|c|c|c|}
\hline Name & Wohnort & Schule & Klasse \\
\hline $\begin{array}{l}\text { Armbruster, } \\
\text { Alexander }\end{array}$ & Unterhaching & $\begin{array}{l}\text { Lise-Meitner- } \\
\text { Gymnasium }\end{array}$ & 12 \\
\hline $\begin{array}{l}\text { Börger, } \\
\text { Christoph }\end{array}$ & Wentorf bei Hamburg & $\begin{array}{l}\text { Gymnasium Wen- } \\
\text { torf }\end{array}$ & 12 \\
\hline $\begin{array}{l}\text { Fronhöfer, } \\
\text { Christoph }\end{array}$ & Landshut & $\begin{array}{l}\text { Hans-Leinberger- } \\
\text { Gymnasium }\end{array}$ & 12 \\
\hline $\begin{array}{l}\text { Groß, } \\
\text { Lukas Finn }\end{array}$ & Essen & $\begin{array}{l}\text { Alfred-Krupp- } \\
\text { Schule }\end{array}$ & 11 \\
\hline $\begin{array}{l}\text { Holstermann, } \\
\text { Jan }\end{array}$ & Warendorf & $\begin{array}{l}\text { Gymnasium Lau- } \\
\text { rentianum }\end{array}$ & 12 \\
\hline $\begin{array}{l}\text { Walter, } \\
\text { Jonas }\end{array}$ & Rostock & $\begin{array}{l}\text { Käthe-Kollwitz- } \\
\text { Gymnasium }\end{array}$ & 11 \\
\hline
\end{tabular}

Alle sechs Teilnehmer haben schon an vielen MathematikWettbewerben teilgenommen. Für Jonas Walter und Alexander Armbruster war es nach Rio de Janeiro 2017 die zweite Teilnahme an einer IMO und die dritte Teilnahme an den IMO-Vorbereitungslehrgängen. Christoph Börger und Jan Holstermann haben auch schon im letzten Jahr an der IMO-Vorbereitung teilgenommen. Auf der diesjährigen Bundesrunde der 57. Mathematik-Olympiade in Würzburg erzielte Jonas Walter mit voller Punktzahl einen 1. Preis in Klasse 11 und die vier Abiturienten erreichten drei 2. und einen 3. Preis und belegten den 2.-4. und den 6. Platz. Alle vier Abiturienten wollen im Wintersemester ein Mathematik-Studium beginnen.

\section{Auswahl und Vorbereitung der deutschen Mannschaft}

Die Auswahl und Vorbereitung der deutschen Mannschaft verlief nach dem Verfahren der Vorjahre. Es qualifizierten sich 76 Schüler und 19 Schülerinnen durch die erfolgreiche Teilnahme an der 2. Runde des Bundeswettbewerbs Mathematik oder an der Bundesrunde der MathematikOlympiaden für zwei Auswahlklausuren, die am 5. und 11. Dezember 2017 geschrieben wurden. Teilgenommen an den Auswahlklausuren haben 70 Schüler und 13 Schülerinnen. Die 16 erfolgreichsten Klausurteilnehmerinnen und Klausurteilnehmer bildeten den Kandidatenkreis für die deutsche Mannschaft. Für diese 14 Schüler und zwei
Schülerinnen gab es Seminare über eine knappe Woche in Rostock, drei Wochenenden in Bad Homburg (jeweils drei Tage) und die traditionelle Abschlusswoche am Mathematischen Forschungsinstitut in Oberwolfach. Während dieser Zeit wurden von allen Kandidaten insgesamt sieben Klausuren geschrieben. Die sechs Besten qualifizierten sich für die IMO-Mannschaft (s. Tabelle 1), deren Zusammensetzung am 17. Mai in Oberwolfach verkündet wurde.

Die Seminare wurden von den Mentoren PD Dr. C. Bey (U Lübeck), Prof. Dr. M. Dreher (U Rostock), Dr. M. Härterich (Wiesloch), Prof. Dr. J. Jahnel (U Siegen), Prof. Dr. U. Leck (U Flensburg), Dr. E. Müller (VillingenSchwenningen), Prof. Dr. J. Prestin (U Lübeck), Dr. C. Reiher (U Hamburg), A. Riekert (U Bonn), G. Schröter (Osnabrück) und F. Schweiger (U Bonn) geleitet.

Zusätzlich hat die Mannschaft vom 20. bis 24. Juni und vom 1 . bis 4 . Juli wieder zwei selbstständig gestaltete Trainingswochenenden in Bonn durchgeführt. Bereits in den Vorjahren fanden solche von den Teilnehmern als sehr effektiv erachtete Zusammenkünfte statt. Dieses Training hat sich hervorragend bewährt. Die Hilfestellung der Geschäftsstelle beschränkte sich hier auf die Organisation der Plätze in der Jugendherberge in Bonn.

Erwähnenswert ist wieder das Zusatztraining im Vorfeld dieses Auswahlverfahrens. Dr. E. Müller (VillingenSchwenningen), J. Reinhold, L. Sauermann (beide U Stanford) und F. Schweiger (U Bonn) betreuten in der zweiten Jahreshälfte 2017 per E-Mail-Korrespondenz sehr intensiv sieben erfolgreiche IMO-Teilnehmer, die auch noch in diesem Jahr teilnahmeberechtigt waren. Sechs von ihnen konnten sich in den Dezemberklausuren für die Vorbereitungslehrgänge qualifizieren, vier von ihnen nahmen an der diesjährigen IMO teil.

Seit 2007 gibt es das Programm Jugend trainiert Mathematik (JuMa). Es wurde u. a. zur besseren Vorbereitung unserer Schülerinnen und Schüler auf die IMO initiiert. Viele der erfolgreichen Teilnehmer an den bundesweiten Mathematik-Wettbewerben und auch vier unserer sechs IMO-Teilnehmer wurden durch dieses Projekt gefördert.

Die gesamte organisatorische Vorbereitung und Durchführung der Klausuren, der Seminare und der Reise wurden wiederum von der Geschäftsstelle der bundesweiten Mathematik-Wettbewerbe unter Leitung von H.-H. Langmann (bis Dezember 2017) und P. Bauermann (ab Januar 2018) in gewohnt perfekter Weise abgewickelt. Allen, die an der Organisation und der Vorbereitung des deutschen Teams beteiligt waren, gebührt herzlicher Dank. 


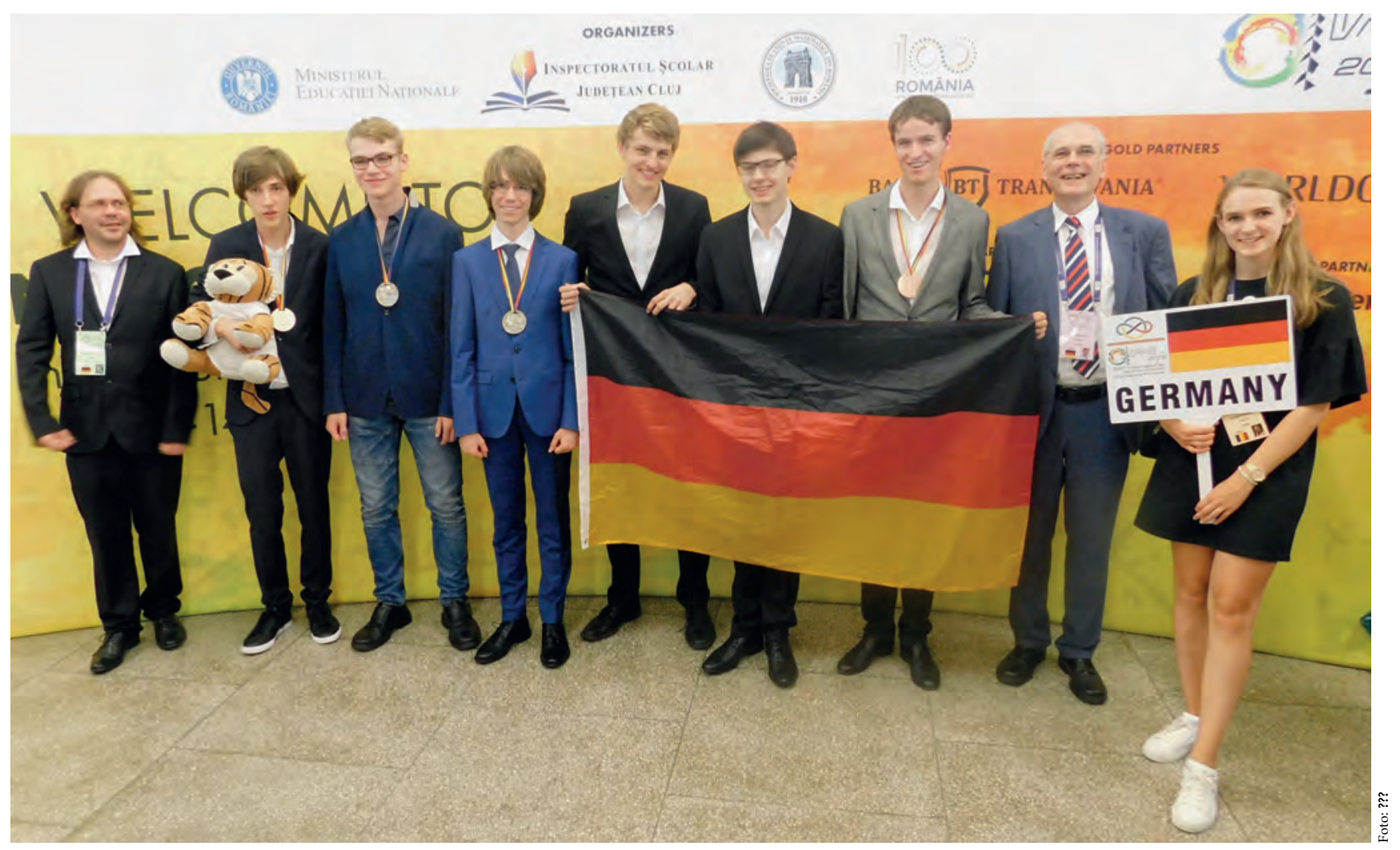

Das deutsche IMO-Team 2018: Dr. Christian Reiher, Jonas Walter, Lukas Finn Groß, Christoph Börger, Jan Holstermann,

Christoph Fronhöfer, Alexander Armbruster, Prof. Dr. Jürgen Prestin, Ilinca Maria Farcaş (Guide) (v. l. n. r.)

\section{Der Ablauf der 59. IMO}

Die Delegationsleiter der Länder, welche die internationale Jury bilden, reisten am 3. und 4. Juli an. Die Jury muss bis zum Ende der Klausuren strikt von den Mannschaften getrennt sein. Allerdings fand in diesem Jahr der oft nach den Klausuren übliche Umzug zu den Schülern nicht statt. Die Delegationsleiter blieben die gesamte Zeit im Grand Hotel Italia in Cluj-Napoca und die stellvertretenden Delegationsleiter kamen für die beiden Tage der Korrektur und des Koordinationsprozesses per Bus-Shuttle in dieses Tagungshotel. Die Schüler und die stellvertretenden Delegationsleiter kamen am 7. Juli in Cluj-Napoca an.

Der Eröffnungsfeier am 8. Juli in einer großen Sporthalle wohnten eine Reihe von Ehrengästen bei. Es gab ausführliche Grußworte von insgesamt ca. 75 Minuten Dauer u. a. vom Präsidenten Rumäniens, einer stellvertretenden Ministerpräsidentin, dem Bildungsminister, dem Bürgermeister von Cluj-Napoca und dem Ehrenpräsidenten dieser IMO, Prof. Viorel Barbu. Prof. Barbu leitet die Mathematik-Abteilung der rumänischen Akademie der Wissenschaften und war selber als Schüler Teilnehmer der 1. IMO 1959 in Braşov. Der Höhepunkt der Veranstaltung war dann wieder die traditionelle Parade aller teilnehmenden Mannschaften über die Bühne.

Am 9. und 10.7. wurden vormittags die beiden $4^{1 / 2-}$ stündigen Klausuren geschrieben. Die Arbeitsbedingungen waren sehr gut. Zur Beantwortung der Schülerfragen in den ersten 30 Minuten der Klausuren konnte die Jury in ihrem Hotel bleiben. Die Fragen wurden eingescannt geschickt und die Antworten auch eingescannt zurückgeschickt. Wieder wurde pro Aufgabe je ein Team von Koordinatoren und Jury-Mitgliedern gebildet, welches die Anfragen mit den jeweiligen Delegationsleitern diskutierte und dann die Antworten genehmigte. Zum einen verlief dieses Verfahren sehr effizient, zum anderen gab es in diesem Jahr erfreulich wenige Anfragen, nur 25 am ersten Tag und 34 am zweiten Tag, so dass alle Schüler die Antworten auf ihre Fragen zeitnah zurückbekamen.

Nach der Durchsicht der Schülerlösungen durch die Delegationsleitungen fand am 11. und 12. Juli die endgültige Festlegung der Bewertung mit den Koordinatoren statt. Das Programmheft listet 91 Koordinatoren auf, fast ausschließlich Rumänen, aber viele von ihnen auch weltweit tätig an bekannten Universitäten. Aus Deutschland war in diesem Jahr niemand als Koordinator beteiligt. Die gesamte Koordination verlief sehr fair und war professionell organisiert. Verbesserungswürdig bleibt für die Zukunft die Beschlussfassung in der Jury über die Marking schemes. Die Koordinatoren legen dazu Entwürfe vor, deren Diskussion in der Jury sicher noch zielorientierter geführt werden kann.

Auf zwei Ausflügen konnten die Schüler Sehenswürdigkeiten kennenlernen. Am 11. Juli besuchten die Schüler Alba Iulia, eine bereits von den Römern gegründete Stadt mit großer Bedeutung für die rumänische Geschichte. Neben einer Besichtigung der Zitadelle blieb den Schülern eine historische Parade nebst Kanonenschüssen in 
bester Erinnerung. Am Folgetag ging es in das 300 Jahre alte Salzbergwerk Turda, das inzwischen in einen riesigen unterirdischen Freizeitpark, sogar mit Riesenrad und mit mehr als 600 ooo jährlichen Besuchern, umfunktioniert wurde. Die Delegationsleiter bzw. die Stellvertreter hatten schon an den Klausurtagen die Möglichkeit, dieses imposante Bergwerk zu bestaunen. Besonders beeindruckte die Schüler, dass sämtliche Bustouren, auch die in ClujNapoca vom Hotel zum Veranstaltungsort, jeweils von mehreren Polizeifahrzeugen eskortiert wurden.

Auch in diesem Jahr wurde von den Veranstaltern die Idee aufgegriffen, bekannte Mathematiker zu Vorträgen einzuladen. Von den drei Beiträgen blieben den Teilnehmern besonders die Vorträge von zwei ehemaligen sehr erfolgreichen rumänischen IMO-Teilnehmern in Erinnerung: Ciprian Manolescu (drei Goldmedaillen 1995-97, jetzt UCLA) berichtete sehr spannend von seiner eigenen Forschung im Bereich der Knotentheorie und Victor Nistor (zwei Goldmedaillen 1978-79, jetzt Université de Lorraine) stellte an zwei mit Nobelpreisen ausgezeichneten Ergebnissen aus den Wirtschaftswissenschaften und der Physik die Rolle von partiellen Differentialgleichungen und ihrer expliziten Lösung heraus.

Die Preisverleihung fand am 13. Juli in der gleichen Sporthalle wie die Eröffnungsveranstaltung statt. Wiederum gab es eine Reihe von Grußworten durch Ehrengäste, u. a. einer stellvertretenden Ministerpräsidentin. Zum zweiten Mal wurden die jeweils besten Teilnehmerinnen der verschiedenen Kontinente auf die Bühne gebeten und mit einem Sonderpreis ausgezeichnet. Dies waren Yolrada Yongpisanpob aus Thailand, Alina Harbuzova aus der Ukraine, Monica Martinez Sanchez aus Peru und Shuyu Jiao aus Botswana. Der Preis soll in Zukunft jährlich vergeben werden und wird benannt nach der Fields-Medaillistin und zweimaligen IMO-GoldmedaillenGewinnerin Maryam Mirzakhani.

Zur Medaillenübergabe der diesjährigen IMO wurden wie üblich Gruppen von Auszuzeichnenden auf die Bühne gebeten: zuerst Bronze, dann Silber und zum Schluss Gold. Zum Abschluss wurde die IMO-Fahne feierlich an den nächsten Veranstalter, das Vereinigte Königreich, übergeben und mit einem Film über Bath die IMO für das nächste Jahr eingeladen. Die Veranstaltung endete mit einer faszinierenden Lasershow in der abgedunkelten Halle und einem überwältigenden Konfettiregen auf die Sitzreihen der Ausgezeichneten.

Im Anschluss an die Preisverleihung gab es abends eine Farewell Party im Grand Hotel Italia. Während man in früheren Jahren meist landesweise zusammensaß, gab es in diesem Jahr zwei Säle, einen für die Schüler und einen für die Delegationsleitungen. In dem einen Saal wurde Wein zum Essen gereicht und vier Tenöre begleiteten den Abend; aus dem anderen Saal schallten laute Discoklänge. So laut, dass unsere deutschen Schüler dann im Hotelfoyer Karten gespielt haben. Die Rückreise am 14. Juli 2018 brachte die Mannschaft im Direktflug nach München, von wo aus dann jeder einzeln weiterfuhr.

Jedes Team wird bei der IMO von einem Guide begleitet. In diesem Jahr war keine Studentin Guide, son- dern eine Schülerin von einem Gymnasium mit DeutschUnterricht, die unsere Mannschaft sehr engagiert betreut hat.

\section{Der Wettbewerb}

An der 59. IMO nahmen 107 Länder mit 594 Schülern teil (vgl. die Ergebnisübersicht auf Seite 212).

Damit konnten die bisherigen Rekorde von 111 Ländern und 615 Teilnehmern im letzten Jahr in Rio de Janeiro nicht eingestellt werden. Die vier Länder weniger in diesem Jahr waren Kuba, Kenia, Liechtenstein und Nicaragua.

Die internationale Jury, bestehend aus den 107 Delegationsleitern und dem rumänischen Chairman Mihail Bălună, begann am Abend des 4. Juli mit ihrer Arbeit. Die Arbeitsbedingungen der Jury in den Konferenzräumen des Grand Hotel Italia waren insgesamt sehr gut. Als besonderen Clou hatten die Veranstalter jedem Jurymitglied für alle Abstimmungen und das Herunterladen von Dokumenten ein Tablet aus rumänischer Produktion zur Verfügung gestellt. Nach einer Eingewöhnungsphase konnte dann sehr effizient in der Jury abgestimmt werden, so dass sofort nach jeder Abstimmung das Ergebnis, je nach Bedarf als geheime oder offene Abstimmung, projiziert wurde.

Jedes teilnehmende Land hat das Recht, Aufgabenvorschläge einzureichen. In diesem Jahr wurden den Veranstaltern 168 Aufgaben aus 49 Ländern zugesandt. Eine Aufgabenkommission des Veranstalters, bestehend aus sechs rumänischen Mitgliedern und den drei weiteren langjährig erfahrenen Mitgliedern Ilya Bogdanov, Pavel Kozhevnikov (beide Moskau) und Géza Kós (Budapest), stellte daraus im Vorfeld der IMO 28 Aufgaben für eine Shortlist zusammen, welche die Grundlage für die Auswahl der Jury bildeten. Von diesen 28 Aufgaben wurden nach längerer Diskussion drei Aufgaben ausgeschlossen. Zwei Vorschläge waren sehr ähnlich zu Aufgaben, die in China bzw. Australien zur Vorbereitung genutzt wurden. Die dritte Aufgabe war wohl kurzzeitig im Einreichungsprozess auf der nur den Delegationsleitern zugänglichen Homepage des Veranstalters allgemein sichtbar gewesen.

Die Jury legte nach intensiven Diskussionen unter Hinzuziehung eines anonym aufgestellten „Beauty-Contests“ schließlich bis zum Abend des 6. Juli sechs der ShortlistAufgaben für die beiden Klausuren fest, wobei die Aufgaben einerseits eine gute Mischung von unterschiedlichen Schwierigkeitsgraden und mathematischen Gebieten sein sollen, andererseits aber auch möglichst keine "Standard“Lösungen zulassen sollen. Ein deutscher Aufgabenvorschlag hat es leider nicht in die Shortlist geschafft. Am Morgen des 7. Juli setzte sich die „English Language Group“ zusammen, um den endgültigen englischen Text der Aufgaben zu wählen, der danach von der Jury wieder bestätigt werden musste. Dort wurden dann Kompromisse geschlossen, z. B. heißt es dann in Aufgabe 1 „parallel oder gleich", da in einigen Sprachen bzw. Schulsystemen „parallel“ auch immer ungleich bedeutet und dann diese 
Schüler versuchen würden, zu zeigen, dass die Geraden nicht identisch sind. Dass in dieser Aufgabe ein Kreisbogen $A B$ nicht als $A B$ bezeichnet wird, hat allerdings einen anderen Grund: Es gibt Sprachversionen und Drucker, in denen dieses Bogensymbol nicht ordentlich wiedergegeben wird. Stark umformuliert wurde die Aufgabe 4. Hier ist die Originalversion aus der Shortlist:

Queenie and Horst play a game on a $20 \times 20$ chessboard. In the beginning the board is empty. In every turn, Horst places a black knight on an empty square in such a way that his new knight does not attack any previous knights. Then Queenie places a white queen on an empty sqare. The game gets finished when somebody cannot move.

Find the maximal positive $K$ such that, regardless of the strategy of Queenie, Horst can put at least $K$ nights on the board.

In der neu formulierten Variante spielt Wissen über Schachfiguren keine Rolle und auch die Ersetzung der Spielernamen durch Amy und Ben war aus deutscher Sicht eher passend.

Nach Beschlussfassung über die englische Variante der Aufgaben wurden die Aufgaben in die weiteren offiziellen Sprachen Deutsch, Französisch, Russisch und Spanisch übersetzt und von der Jury genehmigt. Jeder Schüler und jede Schülerin erhält die Aufgaben in der Muttersprache und einer zweiten Sprache eigener Wahl. Demgemäß übersetzten die entsprechenden Delegationsleiter die Aufgabentexte in die restlichen 56 Sprachen. Auch alle diese Versionen wurden nach Prüfung durch die Jury bestätigt. Insgesamt standen die Aufgaben in 57 Sprachversionen zur Verfügung und sind auf imo-official.org abrufbar.

Bei der Bewertung der Lösungen wurden 36,8 \% der möglichen Punkte vergeben. Dies ist ein sehr durchschnittlicher Wert. Die beiden Extrema der letzten zehn Jahre lagen 2014 bei 38,2\% und 2015 bei 30,9\%. Auch in diesem Jahr war die Aufgabe 3 wieder besonders schwer, so dass sie es mit ihrer erreichten durchschnittlichen Punktzahl in die Liste der schwersten Aufgaben geschafft hat (vgl. Tabelle 2).

$\mathrm{Zu}$ dieser sehr spannenden Aufgabe über antiPascalsche Dreiecke wurde schon wenige Stunden nach Ende der Klausur in Internetforen berichtet, dass Martin Gardner hierüber schon publiziert hat und dass daraufhin vier chinesische Autoren die Existenzfrage abschließend klären konnten (G. J. Chang, M. C. Hu, K. W. Lih and T. C. Shieh, Exact difference triangles. Bull. Inst. Math., Acad. Sin. 5 (1977), 191-197. Siehe auch https://tinyurl.com/yd99r3rr): Ersetzt man in dieser Aufgabe 2018 durch $n$, dann gibt es bis auf Spiegelung insgesamt nur zwei Lösungen für $n=2$, je vier Lösungen für $n=3$ und $n=4$ sowie noch eine Lösung für $n=5$.

In der „Hall of Fame“ aller IMO-Teilnehmer seit 1959 (siehe die Webseite www.mathematik-olympiaden.de oder www.imo-official.org/hall.aspx) gab es an der Spitze keine Veränderungen. In dieser Liste gehören unverändert Lisa
Sauermann, Christian Reiher, Wolfgang Burmeister, Martin Härterich und Peter Scholze zu den besten 16. Auch im exklusiven „Club der IMO-Teilnehmer mit mindestens drei Goldmedaillen“ gab es in diesem Jahr keine Neuaufnahmen.

Das Reglement, das seit vielen Jahren festgeschrieben ist, sieht vor, dass nicht mehr als die Hälfte der Teilnehmer einen Preis erhält und dass die Anzahl der 1., 2. bzw. 3. Preise möglichst das Verhältnis $1: 2: 3$ aufweisen sollte. Die diesjährigen Punktgrenzen sind in Tabelle 3 angegeben. Eine spannende Frage bleibt immer, ob die Jury das Reglement streng auslegt oder eher $\mathrm{zu}$ approximativen Varianten neigt. Wieder wurde darüber anonym in der Jury abgestimmt. Anonym meint hier, dass der Jury gesagt wurde: „Wenn man für $n$ Punkte eine Medaille bekommt, dann werden insgesamt 306 Medaillen vergeben, bei Punktgrenze $n+1$ genau 289 Medaillen." Bei der Juryabstimmung, ob mehr als die laut Reglement möglichen 297 Teilnehmer eine Medaille bekommen sollen und bei der $n$ den Jurymitgliedern noch unbekannt war, wurde die Zweidrittelmehrheit zum Überstimmen des Reglements in diesem Jahr klar verfehlt. Unter den acht Möglichkeiten, die drei Medaillengrenzen festzulegen, wurde dieses Jahr nicht die strengste Möglichkeit gewählt, sondern bei der Grenze von Bronze zu Silber zu Gunsten von Silber entschieden. Hiervon profitierte auch ein deutscher Teilnehmer.

Es gab auch in diesem Jahr keinen Sonderpreis für die besonders elegante Lösung einer Aufgabe.

\section{Die deutsche IMO-Mannschaft}

Das Ergebnis der deutschen Mannschaft mit insgesamt vier Medaillen ist Tabelle $4 \mathrm{zu}$ entnehmen. Besonders gefreut haben wir uns über die Goldmedaille für Jonas Walter. Seit 2008 gab es für Deutschland nur die vier Goldmedaillen von Lisa Sauermann 2008-11. Selbst innerhalb der 48 Goldmedaillenpreisträger gehört Jonas Walter mit 38 Punkten zu den besten acht Teilnehmern der IMO. Nur in Aufgabe 3 fehlte ihm ein Beweisteil, für den vier Punkte im Marking Scheme festgelegt waren. Besonders zu erwähnen ist, dass dies die 50. Goldmedaille einer bundesdeutschen IMO-Mannschaft war. Zur kompletten Statistik nach dieser IMO gehören genau 1 oo Silbermedaillen, 76 Bronzemedaillen und 13 ehrende Erwähnungen für bundesdeutsche Mannschaften.

In der inoffiziellen Länderwertung liegt Deutschland auf Rang 31 und damit um zwei Plätze höher als 2017 in Rio de Janeiro. Hier ist durchaus Verbesserungspotenzial vorhanden, wenn man auf Rang $19 \mathrm{im} \mathrm{Jahr} \mathrm{2016,} \mathrm{davor}$ auf die Ränge 27, 16, 27, 31, 11, 9, 9, 20, 15 und 2006 sogar auf Rang 4 zurückblickt.

Interessant ist wieder ein Blick auf die Ergebnisse bei den einzelnen Aufgaben. Der Vergleich der erreichten Resultate (in Prozent) aller IMO-Teilnehmer, der Schüler der besten 10 Mannschaften sowie der deutschen Mannschaft gibt Aufschluss darüber, wie unsere Schüler die Aufgaben relativ bewältigten, s. Tabelle 5 . Gefreut haben wir uns über das gute Abschneiden bei Aufgabe 4 . 
Tabelle 2. Kleinste durchschnittlich erreichte Punktzahl seit der 1. IMO 1959

\begin{tabular}{crrr}
\hline$\varnothing$ & \multicolumn{1}{c}{ Absolute Punkte } & Aufgabe & Jahr \\
\hline 0,042 & $3 \times 1+1 \times 4+1 \times 5+2 \times 7=26$ & 3 & 2017 \\
0,152 & $40 \times 1+2 \times 2+5 \times 7=79$ & 6 & 2007 \\
$0, \mathbf{1 6 8}$ & $2 \times 1+1 \times 2+10 \times 3+6 \times 4+2 \times 5+1 \times 6+3 \times 7=95$ & 6 & $\mathbf{2 0 0 9}$ \\
$0, \mathbf{1 8 7}$ & $11 \times 1+3 \times 2+3 \times 3+1 \times 5+1 \times 6+8 \times 7=93$ & 6 & 2006 \\
0,251 & $25 \times 1+14 \times 2+2 \times 5+3 \times 6+10 \times 7=151$ & 3 & $\mathbf{2 0 1 6}$ \\
$0, \mathbf{2 6 0}$ & $31 \times 1+9 \times 2+1 \times 6+12 \times 7=139$ & 6 & $\mathbf{2 0 0 8}$ \\
$\mathbf{0 , 2 7 8}$ & $7 \times 1+9 \times 2+14 \times 3+4 \times 4+1 \times 5+11 \times 7=165$ & 3 & $\mathbf{2 0 1 8}$ \\
$0, \mathbf{2 9 4}$ & $24 \times 1+9 \times 2+5 \times 3+4 \times 4+2 \times 5+14 \times 7=181$ & 6 & $\mathbf{2 0 1 7}$ \\
$0, \mathbf{2 9 6}$ & $15 \times 1+6 \times 2+6 \times 3+2 \times 4+6 \times 5+4 \times 6+7 \times 7=156$ & 6 & $\mathbf{2 0 1 3}$ \\
\hline
\end{tabular}

Tabelle 3. Die Punktgrenzen für die Preise

\begin{tabular}{rll}
\hline 48 & Goldmedaillen & für $\geq 31$ Punkte (von 42) \\
98 & Silbermedaillen & für $\geq 25$ Punkte \\
143 & Bronzemedaillen & für $\geq 16$ Punkte \\
138 & Ehrende Erwähnungen & für eine vollständige Lösung \\
289 & Medaillen & bei 594 Teilnehmern \\
\hline
\end{tabular}

Tabelle 4. Die Ergebnisse des deutschen Teams

\begin{tabular}{lcl}
\hline Name & Punkte & Preis \\
\hline Jonas Walter & 38 & Gold \\
Lukas Finn Groß & 28 & Silber \\
Christoph Börger & 25 & Silber \\
Alexander Armbruster & 21 & Bronze \\
Jan Holstermann & 10 & Ehrende Erwähnung \\
Christoph Fronhöfer & 9 & Ehrende Erwähnung \\
\hline
\end{tabular}

\section{Ausblick}

Die gegenwärtige Situation über die Ausrichtung der nächsten IMOs bis 2023 ist in Tabelle 6 angegeben. Neue Ausrichter ab 2024 wurden vom IMO Board noch nicht der Jury zur Bestätigung vorgelegt. Für 2024 und 2025 gibt es allerdings Länder, die überlegen, sich zu bewerben.

\section{IMO Board}

In diesem Jahr fanden turnusgemäß Wahlen zum IMO Board statt. Die neue Zusammensetzung dieses Gremiums ist in Tabelle 7 angegeben.
Tabelle 5. Die Ergebnisse der einzelnen Aufgaben

\begin{tabular}{clrrr}
\hline Aufgabe & Gebiet & $\begin{array}{c}\text { Alle } \\
(\%)\end{array}$ & $\begin{array}{c}\text { Top 10 } \\
(\%)\end{array}$ & $\begin{array}{r}\text { Deutsches Team } \\
(\%)\end{array}$ \\
\hline 1 & Geometrie & 70,5 & 99,8 & 71,4 \\
2 & Algebra & 42,1 & 94,0 & 54,8 \\
3 & Kombinatorik & 4,0 & 21,0 & 7,1 \\
4 & Kombinatorik & 56,6 & 98,8 & 100,0 \\
5 & Zahlentheorie & 38,5 & 90,5 & 57,1 \\
6 & Geometrie & 9,1 & 38,1 & 21,4 \\
Alle & & 36,8 & 73,7 & 52,0 \\
\hline
\end{tabular}

Tabelle 6. Die nächsten IMOs

\begin{tabular}{llll}
\hline Jahr & Land & Ort & Zeitraum \\
\hline 2019 & Vereinigtes Königreich & Bath & 11.-22.07.2019 \\
2020 & Russland & St. Petersburg & \\
2021 & USA & & \\
2022 & Norwegen & & \\
2023 & Japan & & \\
\hline
\end{tabular}

Tabelle 7. Die Mitglieder des IMO-Boards

\begin{tabular}{lc}
\hline & Amtszeit \\
\hline Vorsitzender: Geoff Smith (Vereinigtes Königreich) & bis 2022 \\
Sekretär: Gregor Dolinar (Slowenien) & bis 2020 \\
Mitglied: Nazar Agakhanov (Russland) & bis 2022 \\
Mitglied: Dávid Kunszenti-Kovács (Norwegen) & bis 2020 \\
Mitglied: Yongjin Song (Südkorea) & bis 2022 \\
ex officio IMO 2018: Radu Gologan (Rumänien) & bis 2019 \\
ex officio IMO 2019: Geoff Smith (Vereinigtes König- & bis 2020 \\
reich) & \\
ex officio IMO 2020: Nazar Agakhanov (Russland) & bis 2021 \\
\hline
\end{tabular}

\section{IMO-Informationen}

Für weitere Informationen zu mathematischen Schülerwettbewerben sei auf die Webseite www. mathematik-olympiaden.de verwiesen. Speziell zu den IMOs sind folgende Webseiten empfehlenswert: www.imo-official.org www.mathematik-olympiaden.de/moev/index.php/ olympiaden/imo

Auch Datenschutz wird inzwischen sehr ernst genommen: Ehemalige Teilnehmer, die namentlich auf den Seiten von imo-official.org gelistet sind, können verlangen, dass ihr namentlicher Eintrag ersetzt wird durch beispielsweise "deutscher Teilnehmer Nr. 2". 


\section{Tag}

1. Es sei $\Gamma$ der Umkreis eines spitzwinkligen Dreiecks $A B C$. Punkte $D$ und $E$ liegen so auf den Strecken $A B$ bzw. $A C$, dass $A D=A E$ gilt. Die Mittelsenkrechten der Strecken $B D$ und $C E$ schneiden die kürzeren Kreisbögen $A B$ bzw. $A C$ von $\Gamma$ in den Punkten $F$ bzw. G. Man beweise, dass die Geraden $D E$ und $F G$ parallel oder gleich sind.

(Griechenland)

2. Man bestimme alle ganzen Zahlen $n \geq 3$, für die reelle Zahlen $a_{1}, a_{2}, \ldots, a_{n+2}$ existieren, so dass $a_{n+1}=a_{1}, a_{n+2}=a_{2}$ und

$$
a_{i} a_{i+1}+1=a_{i+2}
$$

für $i=1,2, \ldots, n$ gelten.

(Slowakei)

3. Ein anti-Pascalsches Dreieck ist eine gleichseitig dreieckige Anordnung von Zahlen in der, mit Ausnahme der Zahlen in der untersten Zeile, jede Zahl gleich dem Absolutbetrag der Differenz der beiden unmittelbar darunter stehenden Zahlen ist. Zum Beispiel ist die folgende Anordnung ein anti-Pascalsches Dreieck mit vier Zeilen, das jede ganze Zahl von 1 bis 10 enthält.

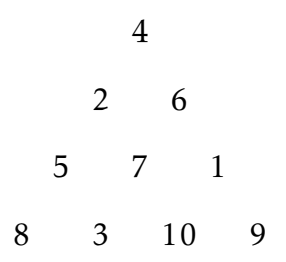

Existiert ein anti-Pascalsches Dreieck mit 2018 Zeilen, das jede ganze Zahl von 1 bis $1+2+\ldots+2018$ enthält?

(Iran)
2. $\mathrm{Tag}$

4. Ein Knoten ist ein Punkt $(x, y)$ in der Ebene, für den sowohl $x$ als auch $y$ positive ganze Zahlen kleiner oder gleich 20 sind.

Zunächst ist jeder der 400 Knoten unbesetzt. Amy und Ben legen abwechselnd Steine auf die Knoten, wobei Amy beginnt. In jedem Zug von Amy legt sie einen neuen roten Stein so auf einen unbesetzten Knoten, dass der Abstand zwischen je zwei von roten Steinen besetzten Knoten ungleich $\sqrt{5}$ ist. In jedem Zug von Ben legt er einen neuen blauen Stein auf einen unbesetzten Knoten. (Ein Knoten, der von einem blauen Stein besetzt ist, darf einen beliebigen Abstand von jedem anderen besetzten Knoten haben.) Sie hören auf, sobald ein Spieler keinen Stein mehr legen kann.

Man bestimme das größte $K$, so dass Amy sicher mindestens $K$ rote Steine legen kann, unabhängig davon, wie Ben seine blauen Steine legt.

(Armenien)

5. Es sei $a_{1}, a_{2}, \ldots$ eine unendliche Folge positiver ganzer Zahlen. Es sei angenommen, dass eine ganze Zahl $N>1$ existiert, so dass für jedes $n \geq N$ die Zahl

$$
\frac{a_{1}}{a_{2}}+\frac{a_{2}}{a_{3}}+\cdots+\frac{a_{n-1}}{a_{n}}+\frac{a_{n}}{a_{1}}
$$

ganz ist. Man beweise, dass es eine positive ganze Zahl $M$ gibt, so dass $a_{m}=a_{m+1}$ für alle $m \geq M$ gilt.

(Mongolei)

6. Ein konvexes Viereck $A B C D$ erfülle die Bedingung $A B \cdot C D=B C \cdot D A$. Ein Punkt $X$ liege so im Inneren von $A B C D$, dass

$$
\Varangle X A B=\Varangle X C D \quad \text { und } \quad \Varangle X B C=\Varangle X D A .
$$

Man beweise, dass $\Varangle B X A+\Varangle D X C=180^{\circ}$ gilt.

(Polen)

Arbeitszeit: $4^{1 / 2}$ Stunden an jedem Tag.

Bei jeder Aufgabe waren 7 Punkte erreichbar. 


\begin{tabular}{|c|c|c|c|c|c|}
\hline $\mathrm{N}$ & Land & $\mathrm{P}$ & G & S & B \\
\hline 1 & USA & 212 & 5 & 1 & - \\
\hline 2 & Russland & 201 & 5 & 1 & - \\
\hline 3 & Volksrepublik China & 199 & 4 & 2 & - \\
\hline 4 & Ukraine & 186 & 4 & 2 & - \\
\hline 5 & Thailand & 183 & 3 & 3 & - \\
\hline 6 & Taiwan & 179 & 3 & 1 & 2 \\
\hline 7 & Republik Korea & 177 & 3 & 3 & - \\
\hline 8 & Singapur & 175 & 2 & 3 & 1 \\
\hline 9 & Polen & 174 & 1 & 5 & - \\
\hline 10 & Indonesien & 171 & 1 & 5 & - \\
\hline 11 & Australien & 169 & 2 & 3 & 1 \\
\hline 12 & Vereinigtes Königreich & 161 & 1 & 4 & - \\
\hline \multirow[t]{2}{*}{13} & Japan & $15^{8}$ & 1 & 3 & 2 \\
\hline & Serbien & $15^{8}$ & 2 & 2 & 2 \\
\hline 15 & Ungarn & 157 & - & 4 & 2 \\
\hline 16 & Kanada & 156 & - & 5 & 1 \\
\hline 17 & Italien & 154 & - & 4 & 2 \\
\hline 18 & Kasachstan & $15^{1}$ & - & 4 & 2 \\
\hline 19 & Islamische Republik Iran & 150 & 1 & 3 & 1 \\
\hline 20 & Vietnam & 148 & 1 & 2 & 3 \\
\hline 21 & Bulgarien & 146 & 1 & 3 & 1 \\
\hline 22 & Kroatien & 145 & - & 4 & 1 \\
\hline 23 & Slowakei & 140 & - & 3 & 3 \\
\hline \multirow[t]{2}{*}{24} & Schweden & 138 & 1 & 2 & 2 \\
\hline & Türkei & 138 & 1 & 1 & 4 \\
\hline 26 & Israel & 136 & - & 2 & 4 \\
\hline 27 & Georgien & 133 & - & 1 & 5 \\
\hline \multirow[t]{3}{*}{28} & Brasilien & 132 & 1 & - & 4 \\
\hline & Indien & 132 & - & 3 & 2 \\
\hline & Mongolei & 132 & - & 1 & 5 \\
\hline 31 & Deutschland & 131 & 1 & 2 & 1 \\
\hline 32 & Armenien & 130 & - & 2 & 4 \\
\hline \multirow[t]{2}{*}{33} & Frankreich & 129 & 1 & 1 & 4 \\
\hline & Rumänien & 129 & 1 & 1 & 2 \\
\hline 35 & Peru & 125 & - & 2 & 3 \\
\hline \multirow{2}{*}{36} & Mexiko & 123 & - & 1 & 4 \\
\hline & Niederlande & 123 & - & 1 & 4 \\
\hline 38 & Philippinen & 121 & 1 & 1 & 2 \\
\hline \multirow[t]{2}{*}{39} & Argentinien & 115 & - & 1 & 4 \\
\hline & Tschechische Republik & 115 & - & 2 & 2 \\
\hline 41 & Bangladesch & 114 & 1 & - & 3 \\
\hline 42 & Slowenien & 104 & - & 1 & 1 \\
\hline \multirow[t]{2}{*}{43} & Bosnien und Herzegowina & 103 & - & - & 4 \\
\hline & Tadschikistan & 103 & - & - & 5 \\
\hline \multirow[t]{2}{*}{45} & Neuseeland & 102 & - & 1 & 2 \\
\hline & Weißrussland & 102 & - & - & 4 \\
\hline 47 & Belgien & 92 & - & - & 4 \\
\hline 48 & Malaysia & 90 & - & - & 2 \\
\hline 49 & Hongkong & 89 & - & - & 2 \\
\hline 50 & Moldawien & 86 & - & - & 3 \\
\hline $5^{1}$ & Estland & 80 & - & 1 & - \\
\hline \multirow[t]{2}{*}{$5^{2}$} & Litauen & 77 & - & - & 2 \\
\hline & Portugal & 77 & - & - & 2 \\
\hline 54 & Griechenland & 74 & - & - & 2 \\
\hline
\end{tabular}

\begin{tabular}{|c|c|c|c|c|c|}
\hline $\mathrm{N}$ & Land & $\mathrm{P}$ & G & $S$ & B \\
\hline 55 & Spanien & 74 & - & - & 2 \\
\hline $5^{6}$ & Norwegen & 73 & - & - & 2 \\
\hline 57 & Österreich & $7^{2}$ & - & - & 3 \\
\hline $5^{8}$ & Dänemark & 71 & - & - & 3 \\
\hline 59 & Finnland & 70 & - & - & 2 \\
\hline \multirow[t]{2}{*}{60} & Saudi-Arabien & 69 & - & 1 & 1 \\
\hline & Syrien & 69 & - & - & 2 \\
\hline 62 & Südafrika & 66 & - & - & 1 \\
\hline \multirow[t]{2}{*}{63} & Costa Rica (5) & 65 & - & - & 2 \\
\hline & Turkmenistan & 65 & - & - & 1 \\
\hline 65 & Macao & 61 & - & - & 1 \\
\hline 66 & Kolumbien & 59 & - & - & 1 \\
\hline 67 & Island & 56 & - & - & 1 \\
\hline 68 & Schweiz & $5^{2}$ & - & - & 1 \\
\hline 69 & Aserbaidschan & 50 & - & - & - \\
\hline 70 & Tunesien & 49 & - & - & - \\
\hline 71 & Ecuador & 48 & - & - & - \\
\hline $7^{2}$ & Sri Lanka & 47 & - & - & 1 \\
\hline \multirow[t]{2}{*}{73} & Marokko & 46 & - & - & - \\
\hline & Puerto Rico & 46 & - & - & 1 \\
\hline 75 & Republik Zypern (5) & 45 & - & - & 1 \\
\hline 76 & Irland & 43 & - & - & 1 \\
\hline 77 & Kirgisistan & 41 & - & - & - \\
\hline 78 & Lettland & 40 & - & - & - \\
\hline 79 & Albanien & 37 & - & - & - \\
\hline 80 & Pakistan & 35 & - & - & - \\
\hline 81 & Bolivien & 33 & - & - & - \\
\hline 82 & Ehem. Jug. Rep. Mazedonien & 27 & - & - & - \\
\hline \multirow[t]{2}{*}{83} & Nigeria $(3)$ & 26 & - & - & - \\
\hline & Trinidad und Tobago (3) & 26 & - & - & - \\
\hline 85 & Myanmar & 23 & - & - & - \\
\hline \multirow[t]{3}{*}{86} & Kosovo & 21 & - & - & - \\
\hline & Panama (4) & 21 & - & - & - \\
\hline & Usbekistan (3) & 21 & - & - & - \\
\hline \multirow[t]{2}{*}{89} & El Salvador (2) & 20 & - & - & - \\
\hline & Montenegro (4) & 20 & - & - & - \\
\hline 91 & Chile (4) & 19 & - & - & - \\
\hline 92 & Algerien (4) & 18 & - & - & - \\
\hline 93 & Luxemburg (2) & 14 & - & - & - \\
\hline 94 & Ghana (5) & 13 & - & - & - \\
\hline \multirow[t]{2}{*}{95} & Botswana & 12 & - & - & - \\
\hline & Paraguay & 12 & - & - & - \\
\hline \multirow[t]{2}{*}{97} & Guatemala (3) & 11 & - & - & - \\
\hline & Kambodscha & 11 & - & - & - \\
\hline 99 & Ägypten (4) & 10 & - & - & - \\
\hline \multirow[t]{2}{*}{100} & Irak & 9 & - & - & - \\
\hline & Uganda (4) & 9 & - & - & - \\
\hline 102 & Côte d'Ivoire & 8 & - & - & - \\
\hline 103 & Uruguay (4) & 7 & - & - & - \\
\hline 104 & Honduras (3) & 6 & - & - & - \\
\hline 105 & Nepal & 5 & - & - & - \\
\hline 106 & Venezuela (1) & 2 & - & - & - \\
\hline 107 & Tansania (3) & 1 & - & - & - \\
\hline
\end{tabular}

Legende: N - Platzierung, P - Punktzahl, G - Anzahl der Goldmedaillen, S - Anzahl der Silbermedaillen, B - Anzahl der Bronzemedaillen. Jede Mannschaft bestand aus sechs bzw. der in Klammern angegebenen Anzahl von Schülerinnen und Schülern. Eine vollständige Mannschaft (sechs Personen) konnte maximal 252 Punkte erreichen. 